\title{
MACC1 overexpression in carcinoma-associated fibroblasts induces the invasion of lung adenocarcinoma cells via paracrine signaling
}

\author{
ZHUOSHI LI ${ }^{1,2^{*}}$, TAO GUO ${ }^{1,2^{*}}$, LEI FANG ${ }^{1,2^{*}}, \mathrm{NAN} \mathrm{LI}^{3 *}$, XIAOCHAO WANG ${ }^{3}$, PENG WANG ${ }^{1,2}$, SHILEI ZHAO $^{1,2}$, \\ FENGZHOU LI ${ }^{1,2}$, YANWEI CUI ${ }^{1,2}$, XIN SHU ${ }^{1,2}$, LEI ZHAO ${ }^{1,2}$, JINXIU LI ${ }^{2}$ and CHUNDONG GU ${ }^{1,2}$ \\ ${ }^{1}$ Department of Thoracic Surgery; ${ }^{2}$ Lung Cancer Diagnosis and Treatment Center of Dalian, \\ The First Affiliated Hospital of Dalian Medical University, Dalian, Liaoning 116011; \\ ${ }^{3}$ Institute of Cancer Stem Cell, Dalian Medical University, Dalian, Liaoning 116044, P.R. China
}

Received January 9, 2018; Accepted November 26, 2018

DOI: $10.3892 /$ ijo.2019.4702

\begin{abstract}
Carcinoma-associated fibroblasts (CAFs) are essential for initiating lung cancer cell invasion and metastasis. An elevated MACC1 expression has been implicated in the progression of lung adenocarcinoma. Hitherto, the role of $\mathrm{MACC} 1$ in lung adenocarcinoma-derived CAFs remains unclear. In this study, CAFs isolated from the tissues of patients with lung adenocarcinoma expressed typical CAF markers (shown by immunohistochemical and immunofluorescence analysis) and exhibited enhanced migration and invasion abilities when co-cultured with A549 cells in a microfluidic model. MACC1-overexpressing CAFs not only demonstrated an increased invasion, but also exerted a promoting effect on the invasion of tumor cells. The reduced expression of MACC1 impaired the invasive ability of the CAFs. Western blot analysis and RT-qPCR analysis demonstrated that multiple paracrine pathways were activated in the MACC1-overexpressing CAFs. Overall, this study presents a novel role of MACC1 in CAF-induced lung adenocarcinoma cell invasion, which possibly occurs via paracrine signaling. Furthermore, MACC1
\end{abstract}

Correspondence to: Professor Chundong Gu, Department of Thoracic Surgery, The First Affiliated Hospital of Dalian Medical University, 222 Zhongshan Road, Dalian, Liaoning 116011, P.R. China

E-mail: guchundong@dmu.edu.cn

Professor Jinxiu Li, Lung Cancer Diagnosis and Treatment Center of Dalian, The First Affiliated Hospital of Dalian Medical University, Dalian, Liaoning 116011, P.R. China

E-mail: lijinxiu@dmu.edu.cn

*Contributed equally

Key words: metastasis-associated in colon cancer-1, carcinoma-associated fibroblasts, microfluidic chip, lung adenocarcinoma, invasion was indicated to be a potential therapeutic target for lung adenocarcinoma.

\section{Introduction}

Lung cancer is the leading cause of cancer-related mortality worldwide (1). Non-small cell lung cancer (NSCLC) accounts for $\sim 85 \%$ of lung cancer cases, with adenocarcinoma being the most common subtype, followed by squamous cell carcinoma (2). The majority of patients with NSCLC are not diagnosed within the optimal time frame and thus cannot undergo successful radical resection due to tumor invasion and distant metastasis (3). The identification of novel potential therapeutic targets which may be used to prevent the invasion of lung adenocarcinoma is urgently required.

Tumor growth and metastasis are closely associated with the properties of tumor cells, as well as with the tumor stroma $(4,5)$. Fibroblasts in the tumor stroma are permanently activated and serve as important precursors of tumor growth, invasion and metastasis (6-9). Cancer-associated fibroblasts (CAFs) express $\alpha$-smooth muscle actin ( $\alpha$-SMA) and vimentin (VIM), and exhibit a high degree of heterogeneity $(4,5,10)$. However, cytokeratin (CK) derived from epithelium-originated cancer is not expressed in CAFs (11). CAFs promote tumor progression by secreting chemokines, and other factors in various types of cancer $(4,12-14)$. A recent study suggested that CAFs enhance the invasive ability of lung adenocarcinoma cells (15). However, the underlying mechanisms remain unclear.

Metastasis-associated in colon cancer-1 (MACC1) was originally identified in primary and metastatic colon carcinomas (16). MACC1 expression has been demonstrated to be upregulated in different types of cancer (17). It has also been indicated that MACC1 is involved in tumor invasion and metastasis in gastric cancer (18), breast cancer (19) and hepatocellular cancer $(20,21)$. It was previously shown that MACC1 was overexpressed in lung adenocarcinoma tissue, and was associated with the rate of tumor metastasis and invasion $(22,23)$. However, the role of MACC1 in CAFs, particularly as regards the promoting effects on cancer cell 
invasive ability, remains poorly characterized. Therefore, in the present study, the effects and potential mechanisms of action of MACC1 as regards tumor invasion in lung adenocarcinoma were investigated.

\section{Materials and methods}

Cell lines. The human lung cancer cell line (A549), the normal human fetal lung fibroblast cell line (HFL-1) and human embryonic kidney 293T cell line were purchased from the American Type Culture Collection (ATCC, Manassas, VA, USA).

Patients and tissue specimens. The present study was approved by the Ethics Committee of the First Affiliated Hospital of Dalian Medical University (Ethics no. YJ-KY-FB-20). Tissue samples were collected from 7 patients with lung adenocarcinoma who underwent radical surgery of the primary tumor and systematic nodal dissection without any adjuvant therapy at the Department of Thoracic Surgery of the First Affiliated Hospital of Dalian Medical University from January, 2017 to March, 2017. All the patients had signed an informed consent form. The included patients (Table I) were aged between 58 to 76 years (median age, 64 years).

Primary cell culture. The fresh specimens sectioned into a smooth paste were digested with $5 \mathrm{mg} / \mathrm{ml}$ collagenase I and $0.2 \mathrm{mg} / \mathrm{ml}$ hyaluronic acid dissolved into Dulbecco's modified Eagle's medium (DMEM; Thermo Fisher Scientific, Waltham, MA, USA) plus $10 \%$ fetal bovine serum (FBS; Thermo Fisher Scientific) in a $37^{\circ} \mathrm{C}$ incubator for $5 \mathrm{~h}$. Tissue debris was removed by a filter and cells were collected and cultured in a $37^{\circ} \mathrm{C}$ humidified atmosphere containing $95 \%$ air and $5 \% \mathrm{CO}_{2}$. Once the cells reached $80-90 \%$ confluence, they were harvested and reseeded in complete medium (DMEM plus $10 \%$ fetal bovine serum).

Immunohistochemical staining (IHC). The lung tissue specimens were first formalin-fixed and paraffin-embedded, rehydrated and incubated in 3\% (v/v) hydrogen peroxide (Sigma-Aldrich, St. Louis, MO, USA) for $10 \mathrm{~min}$. Antigenic retrieval was processed with sodium citrate. IHC staining was performed using the Streptavidin-Peroxidase IHC assay kit (ZSGB-Bio, Beijing, China) following the manufacturer's instructions. The antibody to $\alpha$-SMA (rabbit; cat. no. ab5694; Abcam, Cambridge, MA, USA) was diluted 1:200 proportionally in PBS containing $2 \%$ goat bovine serum. Immunostaining was evaluated by two pulmonary pathologists using a blinded protocol design.

Immunofluorescence. The grown CAFs isolated from the tumor tissues were seeded on coverslips within 6-well plates. were fixed with $4 \%$ paraformaldehyde, permeabilized with $0.2 \%$ Triton X-100, and then blocked with $10 \%$ bovine serum albumin. The primary antibodies against pan-ck (mouse; ZSGB-Bio; diluted 1:100), $\alpha$-SMA (rabbit; cat. no. ab5694; diluted 1:100; Abcam) and Vimentin (rabbit; cat. no. D21H3; diluted 1:200; Cell Signaling Technology, Danvers, MA, USA) and diluted in blocking solution. Immune complexes were stained with the secondary antibody conjugated to Alexa-488 (cat. no. A-11008; diluted 1:100; Thermo Fisher Scientific) or
Alexa-546 (cat. no. A10040; diluted 1:100; Thermo Fisher Scientific). The nuclei were stained with DAPI at $25^{\circ} \mathrm{C}$ for 4 min (Sigma-Aldrich). Each experimental group contained 6 wells each time, and 5 fields of each well were imaged using a fluorescence microscope (Olympus IX71; Olympus Corporation, Tokyo, Japan).

Western blot analysis. The cells were collected at the optimum cell culture time in a microcentrifuge tube. Total protein was extracted using RIPA lysing buffer, and lysates were centrifuged at $12,000 \mathrm{x}$ for $10 \mathrm{~min}$ at $4{ }^{\circ} \mathrm{C}$. The concentration of the total, nuclei or cytoplasm cell protein lysate was quantified using a BCA protein assay kit (Thermo Fisher Scientific). Subsequently, 30-50 $\mu \mathrm{g}$ of protein lysates were separated by $10 \%$ SDS-PAGE and transferred onto a polyvinylidene difluoride (PVDF) membrane. The membrane was incubated with the specific antibodies (appropriate concentration referred to specification) and protein bands were detected with an enhanced chemiluminescence system (MilliporeSigma, Burlington, MA, USA). The following antibodies were used: Anti-Vimentin (rabbit; cat. no. D21H3; diluted 1:1,000; Cell Signaling Technology), anti-GAPDH (mouse; diluted 1:5,000; cat. no. SAB1405848; Sigma-Aldrich), anti-MACC1 (rabbit; diluted 1:1,000; cat. no. ab106579; Abcam), anti-hepatocyte growth factor (HGF; diluted 1:1,000; rabbit; cat. no. ab83760; Abcam), anti-leukemia inhibitory factor (LIF; diluted 1:1,000; rabbit; cat. no. 26757-1-AP; Proteintech, Rosemont, IL, USA), anti-CXCL12 (rabbit; diluted 1:1,000; cat. no. 17402-1-AP; Proteintech), anti-insulin-like growth factor 2 (IGF2; diluted 1:1,000; mouse; cat. no. SAB1406010; Sigma-Aldrich), goat-antirabbit $\mathrm{IgG}$ conjugated to horseradish peroxidase (HRP; cat. no. 31460; Thermo Fisher Scientific) and goat-anti-mouse IgG conjugated to HRP (cat. no. 31430; Thermo Fisher Scientific) which was used as the secondary antibody. The densitometry of the blots was calculated using Image lab 3.5 and the bar graph was generated using the Graphpad Prism6.

Reverse transcription-quantitative PCR (RT-qPCR). Total RNA was extracted from the cells using TRIzol reagent (Invitrogen/Thermo Fisher Scientific), which was used to generate cDNA using the PrimeScript RT reagent kit (Takara Bio USA, Inc., Mountain View, CA, USA). The resulting cDNA was analyzed in triplicate using reaction 2 steps qPCR $\left(95^{\circ} \mathrm{C}\right.$ for $30 \mathrm{sec}$, followed by 40 cycles of $95^{\circ} \mathrm{C}$ for $10 \mathrm{sec}$, annealing at $60^{\circ} \mathrm{C}$ for $30 \mathrm{sec}$ and followed by 1 cycle of $95^{\circ} \mathrm{C}$ for $15 \mathrm{sec}$, at $60^{\circ} \mathrm{C}$ for $1 \mathrm{~min}$ and $95^{\circ} \mathrm{C}$ for $15 \mathrm{sec}$ ) with SYBR Select Master Mix (Life Technologies/Thermo Fisher Scientific). The qPCR sequences were listed as follows: ACTB sense, 5'-CATGTACGTTGCTATCCAGGC-3' and antisense, 5'-CTCCTTAATGTCACGCACGAT-3'); MACC1 sense, 5'-TTCTTTTGATTCCTCCGGTGA-3' and antisense, 5'-ACT CTGATGGGCATGTGCTG-3'; HGF sense, 5'-TGCACG ACAGTGTTTCCCTT-3' and antisense, 5'-CACATCCAC GACCAGGAACA-3'; LIF sense, 5'-GTCTTGGCGGCA GTACACAG-3' and antisense, 5'-CGACTATGCGGTACA GCTCC-3'; CXCL12 sense, 5'-CAGATGCCCATGCCGATT CT-3' and antisense, 5'-TTCTTCAGCCGGGCTACAAT-3'; IGF2 sense, 5'-AACTTCCCCAGATACCCCGT-3' and antisense, 5'-GGGTGGGTAGAGCAATCAGG-3'. 
Table I. Characteristics of the patients with pancreatic adenocarcinoma.

\begin{tabular}{lcccccc}
\hline Patient no. & Age (years) & Sex & Metastasis status & Tumor stage & Grade & Pathological subtype \\
\hline $1($ CAF-A) & 58 & F & - & T1N0M0 & 1 & Adenocarcinoma \\
$2($ CAF-B) & 59 & F & - & T1N0M0 & 1 & Adenocarcinoma \\
$3($ CAF-1) & 65 & M & - & T1N0M0 & 1 & Adenocarcinoma \\
$4($ CAF-2) & 63 & M & - & T1N0M0 & 1 & Adenocarcinoma \\
$5($ CAF-3) & 59 & F & - & T1N0M0 & 1 & Adenocarcinoma \\
$6($ CAF-4) & 68 & T & - & T1N0M0 & 1 & Adenocarcinoma \\
$7($ CAF-5) & 76 & $M$ & - & 1 & Adenocarcinoma
\end{tabular}

F, female; M, male.

A
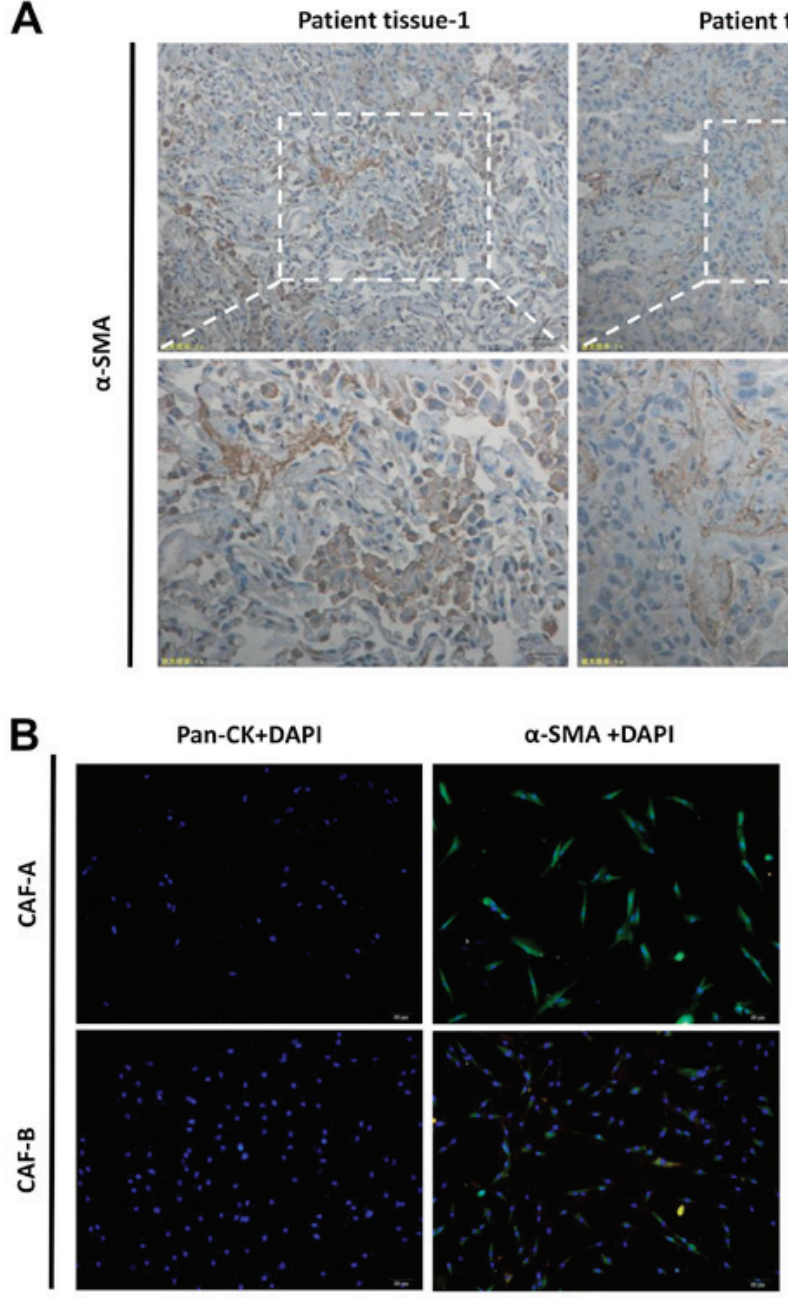

Patient tissue-2

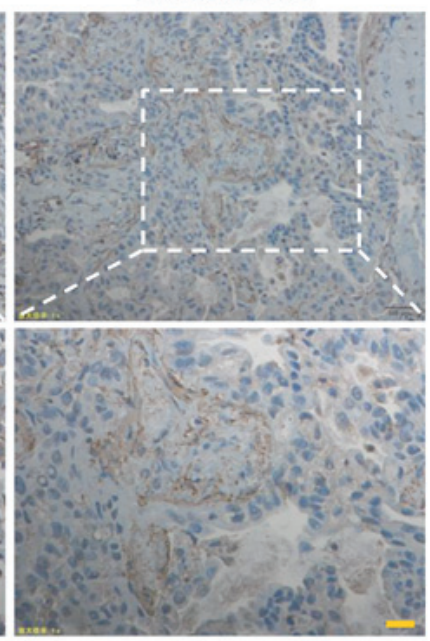

Vimentin +DAPI

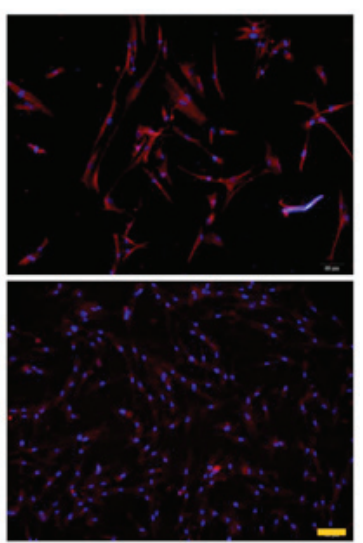

Figure 1. CAFs were detected in activated fibroblasts. (A) Immunohistochemical staining revealed that spicemens from patients with lung adenocarcinoma expressed $\alpha$-SMA. (B) Immunofluorescent staining revealed that CAF-A and CAF-B cells were negative for pan-CK expression and positive for Vimentin and $\alpha$-SMA expression. CAFs, carcinoma-associated fibroblasts; $\alpha$-SMA, $\alpha$-smooth muscle actin.

Plasmid constructs. The plasmid encoding human MACC1 was generated by PCR amplification and inserted into the pLVX-DsRed-N1-Monomer vector (Clontech/Takara Bio USA, Inc.). The primers for gene cloning were as follows: MACC1 5'XhoI, 5'-CACTCGAGGCCACCATGCTAATC ACTGAAAGAAAACATTTTCGG-3'; 3'XmaI, 5'-CACCCG GGCTACTTCCTCAGAAGTGGAGAATGCAGTTACTC-3'.
Lentivirus preparation and transfection. The targeting MACC1 and the non-target shRNA (shMACC1 and shNC) were purchased from GenePharma (Shanghai, China). The target siRNA sequences of MACC1 were as follows: 5'-GUG ACGAGGAAUUGACAAUTT-3', and that of the negative control siRNA was 5'-UUCUCCGAACGUGUCACGUTT-3'). 293T cells were co-transfected with the psPAX2 and pMD2.G 
A

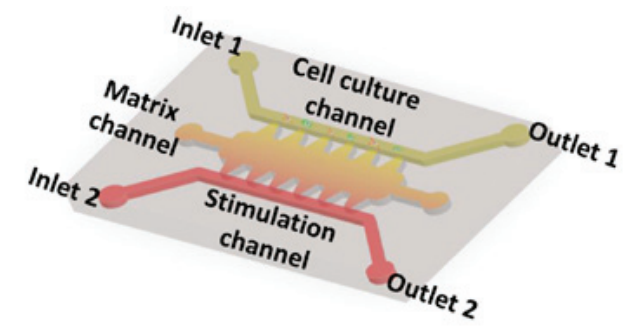

B
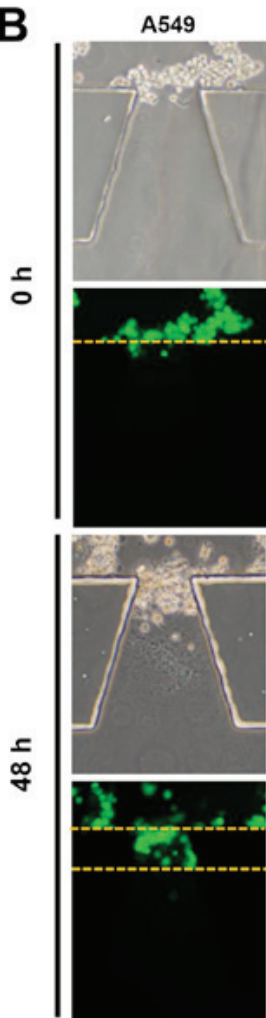

A549+CAF\#1
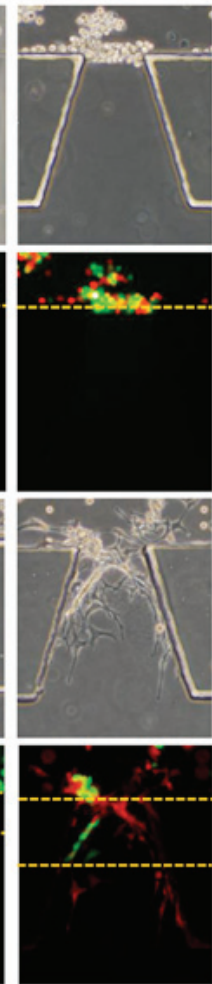

A549+CAF\#II
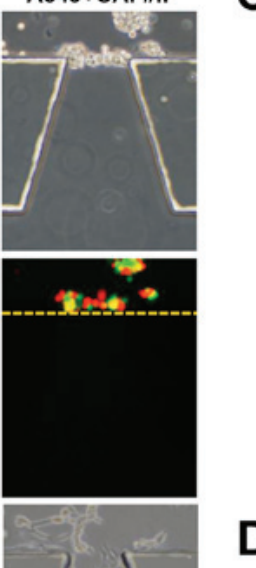

D

C
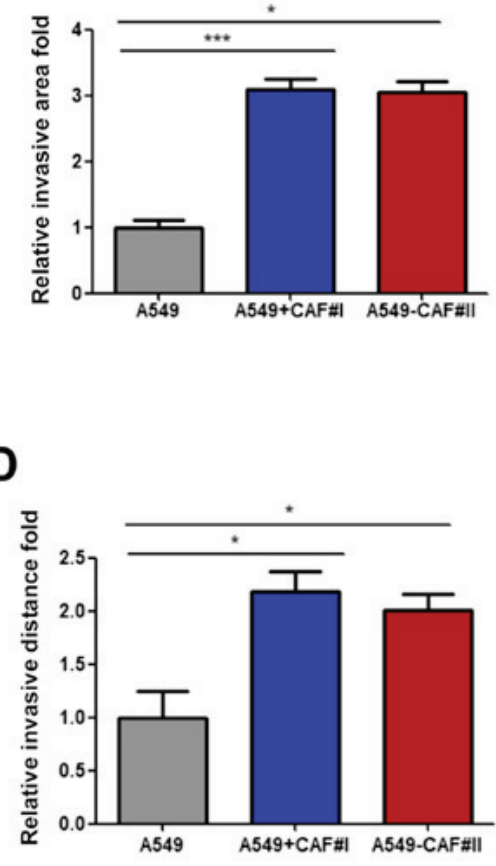

Figure 2. CAFs Promote the invasion of lung adenocarcinoma cells. (A) Diagram of a microfluidic device. (B) The microfluidic model-based cell invasion assay indicated that CAFs increased (C) the invasion area and (D) the invasion distance in the co-culture system of A549 cells and CAFs. Error bars represent the means \pm SD derived from 3 independent experiments. ${ }^{*} \mathrm{P}<0.05$ and ${ }^{* * *} \mathrm{P}<0.005$. CAFs, carcinoma-associated fibroblasts.

using Lipofectamine 2000 (Life Technologies/Thermo Fisher Scientific). Lentiviruses were harvested and concentrated by ultracentrifugation (1,500 rpm at room temperature for $5 \mathrm{~min}$ ) at $48 \mathrm{~h}$ following transfection, and viral titer determined by serial dilutions. Infected cells (A549 and CAFs) were selected with puromycin ( $2 \mathrm{mg} / \mathrm{ml}$; Sigma-Aldrich).

Cell invasion assay based on the microfluidic model. To estimate the invasive potential of the CAFs, a microfluidic device developed in our previous study was used (24). Matrigel, as the substitute for ECM, was loaded into the middle channel of the microfluidic device. The cells (A549, CAF\#I, CAF\#II, CAF-2, CAF-3, CAF-2-VECTOR, CAF-2-MACC1-OE, CAF-shNC and CAF-3-shMACC1) were seeded into the upper channel in serum-free medium for cell culture and medium with $10 \%$ FBS was loaded into the under channel for stimulation. The device was incubated for $48 \mathrm{~h}$ at $37^{\circ} \mathrm{C}$. Cell invasion area was determined as the area invaded by cells in the matrix channel and measured by Image-Pro Plus6.0. For observation, A549 with green fluorescence by infected by pEZ-vector (GeneCopoeia USA, Inc.) and CAF\#I CAF\#II were marked as CAF-A and CAF-B after immunofluorescence assay by infected pLVX-DsRed-N1-Monomer vector (Clontech/Takara Bio USA, Inc.).

Statistical analysis. The Student's t-test and analysis of variance (one-way ANOVA) followed by appropriate post hoc tests (Fishers' Least Significant Difference) were used to compare the values of the test and control samples in vitro. Values of $\mathrm{P}<0.05$ were considered to indicate statistically significant differences.

\section{Results}

Highly active CAFs are detected in patients with lung adenocarcinoma. A number of $\alpha$-SMA-positive cells were observed in the pulmonary mesenchyme by immunohistochemical staining (Fig. 1A), indicating the CAFs in two lung adenocarcinoma cases. CAF-A and CAF-B were extracted from the two lung adenocarcinoma patient tissues, and both cell types exhibited a typical fibroblastic morphology with cytoplasmic processes on a monolayer. They were 

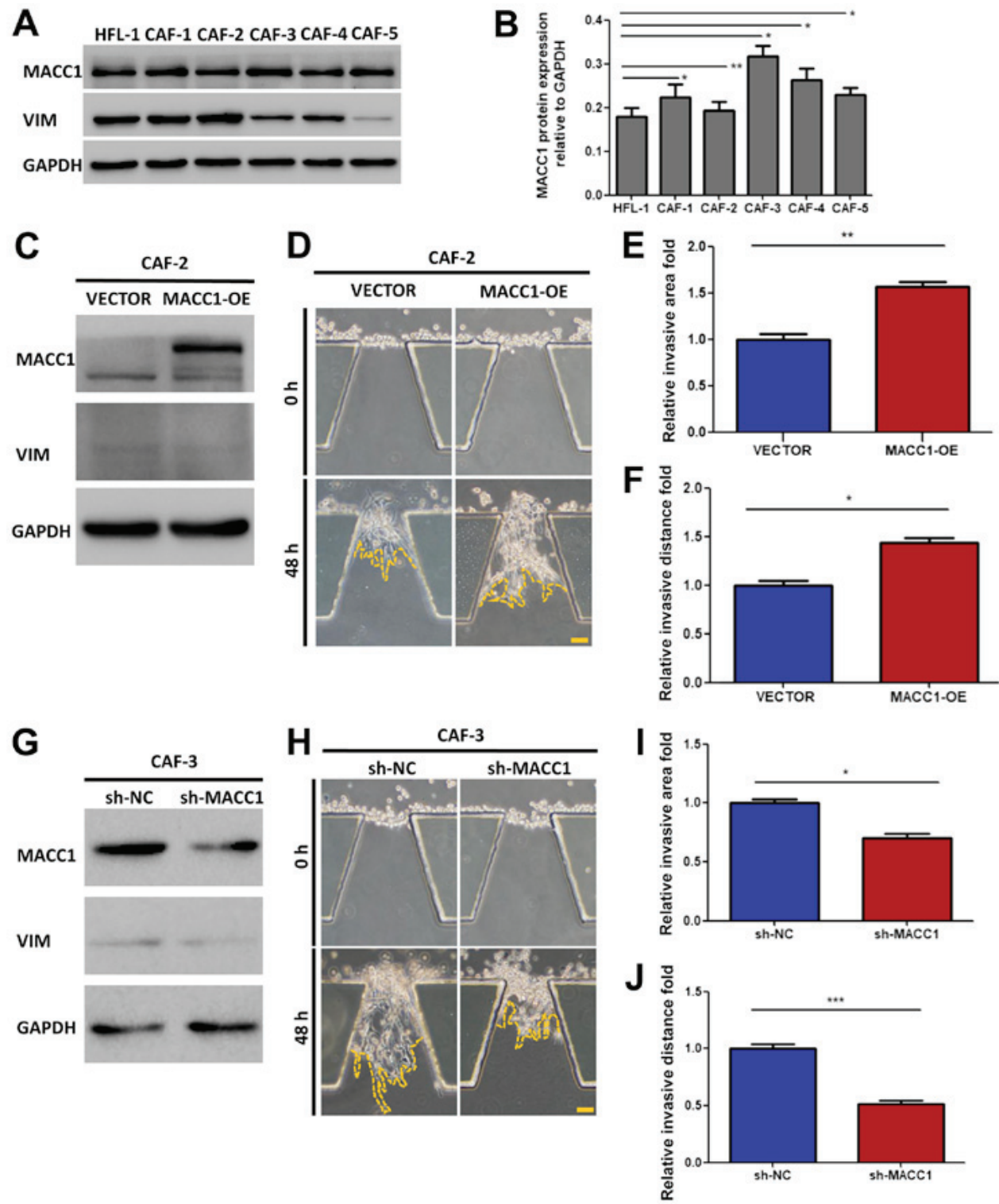

Figure 3. MACC1 promotes the invasive ability of CAFs. (A) Western blot analysis revealed that MACC1 was highly expressed in the CAFs extracted from lung adenocarcinoma tissues compared with normal fibroblasts (HFL-1). (B) MACC1 protein expression was calculated relative to that of GAPDH. (C) Efficiency of transfection was examined by western blot analysis. (D) The microfluidic model-based cell invasion assay revealed that elevated MACC1 expression in CAFs enhanced (E) the invasion area and (F) the invasion distance. (G) Efficiency of MACC1-knockdown was examined by western blot analysis. (H) MACC1 knockdown impaired (I) the invasion area and (J) the invasion distance. Error bars represent the means \pm SD derived from 3 independent experiments. "P<0.05, ${ }^{* *} \mathrm{P}<0.01$ and ${ }^{* * *} \mathrm{P}<0.005$. MACC1, metastasis-associated in colon cancer-1; CAFs, carcinoma-associated fibroblasts.

negative for pan-ck and positive for $\alpha$-SMA and Vimentin. The activated status of the CAFs were detected in the primary cells from the patients with lung adenocarcinoma (Fig. 1B).

CAFs promote the invasion of lung adenocarcinoma cells. Accumulating evidence suggests that the migration and invasion process of tumor cells may be regulated by the function of CAFs (5). In this study, to confirm whether CAFs can promote lung adenocarcinoma cells lateral invasion process beyond co-culture condition, a microfluidic model was employed, as previously described (24). The cell culture, stimulation channel and matrix channels were independent. The device, consisting of a glass slide and translucent PDMS, was sterilized and bonded to a $10-\mathrm{cm}$ dish prior to use (Fig. 2A). The A549 cells were cultured alone as a standard control, or co-cultured with CAF\#I or CAF\#II in a microfluidic chip (Fig. 2B). Matrigel and medium containing $10 \%$ serum was loaded into the matrix channels prior to addition of the separate groups into the cell culture channel into the cell culture channel, and cultured in serum-free medium for $48 \mathrm{~h}$. The invasive distance and area were increased in the co-culture condition, compared with the A549 cells cultured alone (Fig. 2C and D).

MACC1 promotes the invasive ability of CAFs. MACC1 has been demonstrated to promote the metastasis and invasion of lung adenocarcinoma cells (25). In this study, to investigate the role of MACC1 in the invasive process of CAFs, we detected the protein expression level of MACC1 in CAFs isolated from 5 patients with lung adenocarcinoma, and compared the result with normal fibroblast cells (HFL-1) (Fig. 3A and B). MACC1 was expressed in all the CAFs, and the cells exhibiting the lowest and highest expression were selected for use in further 
A

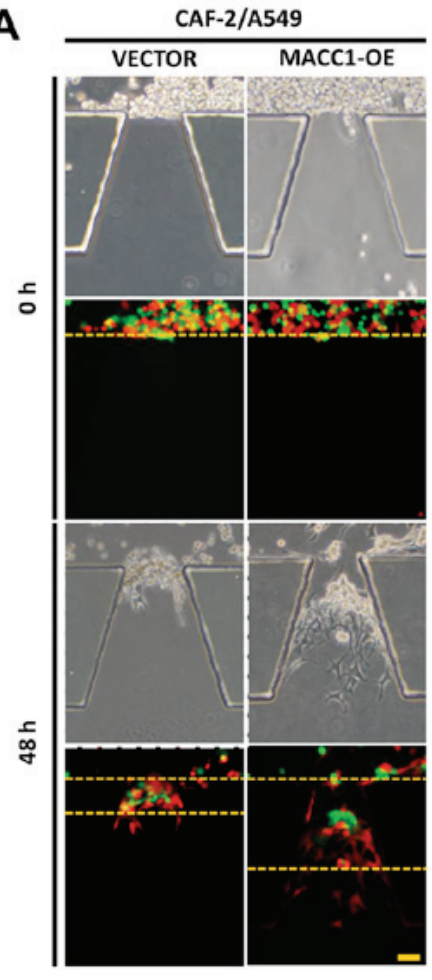

B

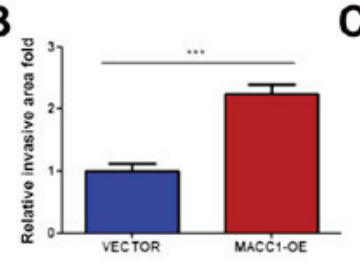

$\mathrm{C}_{\mathrm{g}}$

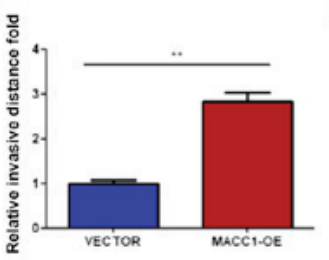

D

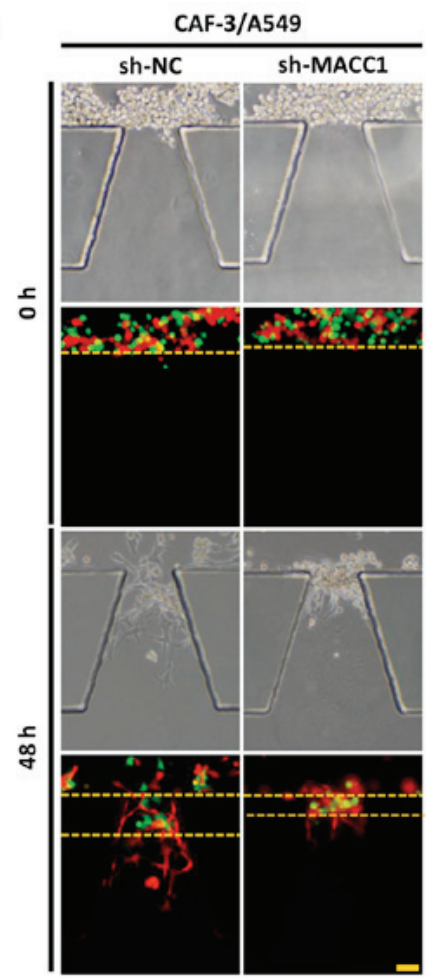

E

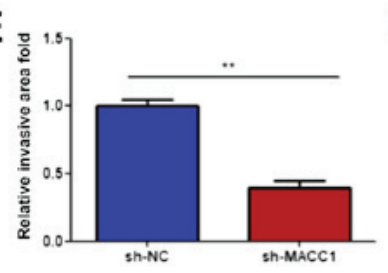

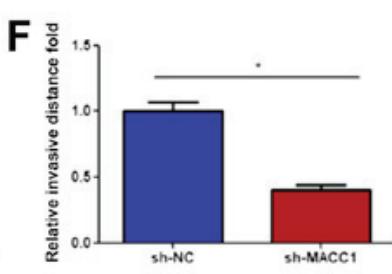

Figure 4. MACC1 overexpression in CAFs stimulates the invasion of A549 cells. The microfluidic model-based cell invasion assay revealed (A) that MACC1-overexpressing CAFs enhanced the invasive ability of lung adenocarcinoma A549 cells by increasing (B) the invaion area and (C) the invasion distance. (D) MACC1 knockdown reduced (E) the invasion area and (F) the invasion distance in the co-culture system. Error bars represent the means \pm SD derived from 3 independent experiments. ${ }^{*} \mathrm{P}<0.05,{ }^{* *} \mathrm{P}<0.01$ and ${ }^{* * *} \mathrm{P}<0.005$. MACC1, metastasis-associated in colon cancer-1; CAFs, carcinoma-associated fibroblasts.

experiments: CAF-2 and CAF-3, respectively. Subsequently, the overexpression of $\mathrm{MACC} 1$ was induced in the CAF-2 cells (Fig. 3C) and it was knocked down in the CAF-3 cells (Fig. 3G). The stably transfected cells (CAF-2-VECTOR, CAF-2-MACC1 or CAF-3-shNC, CAF-3-shMACC1) were injected into microfluidic chips. After $48 \mathrm{~h}$, the cell invasive ability was examined and it was found to be enhanced in the CAF-2 cells (Fig. 3D), as indicated by the increase in both the invasive distance and area, compared with the controls (Fig. 3E and F). By contrast, the invasive ability was markedly reduced in the CAF-3 cells in which MACC1 was knocked down (Fig. $3 \mathrm{H}$ ), as indicated by a decrease in both invasive the distance and area (Fig. 3I and J).

Expression of MACCl by CAFs stimulates the invasion of lung adenocarcinoma cells. As suggested by the above-mentioned results, MACC1 promotes the invasive ability of the CAFs. We investigated whether interfering with MACC1 expression in CAFs can influence the invasive activity of lung adenocarcinoma cells in co-culture conditions. Stably transfected cell lines, CAF-2-VECTOR, CAF-2-MACC1, CAF-3-shNC,CAF-3-shMACC1 were injected with A549 cells into microfluidic chips. After $48 \mathrm{~h}$, the invasive ability of the A549 cells was enhanced by co-culture with CAF-2-MACC1 cells (Fig. 4A-C). However, the invasive ability of the A549 cells was impaired by co-culture with CAF-3-shMACC1 cells (Fig. 4D), as indicated by the changes in the invasive distance and area (Fig. 4E and F).

Overexpression of MACCl induces CAFs to secrete chemokines through paracrine signaling. Stromal-derived IGF2, HGF, LIF and CXCL12 have been demonstrated to promote cancer progression and invasion via paracrine signaling $(4,12-14)$. In this study, we aimed to explore the mechanisms responsible for the enhancement of lung adenocarcinoma cell motility by MACC1, by co-culture with CAFs. In stable MACC1-overexpressing CAF-2 cells, the expression levels of CXCL12, $\operatorname{LIF}(\mathrm{P}<0.05)$ and $\operatorname{HGF}(\mathrm{P}<0.001)$ were increased at the transcriptional and proteinic level compared with the CAF-2-VECTOR cells (Fig. 5A and B). In stable CAF-3-shMACC1 cells, the expression levels of HGF, LIF $(\mathrm{P}<0.05)$ and CXCL12 $(\mathrm{P}<0.01)$ were decreased at the transcriptional and protein level compared with the CAF-3-shNC cells (Fig. 5C and D). It can thus be hypothesized 
A

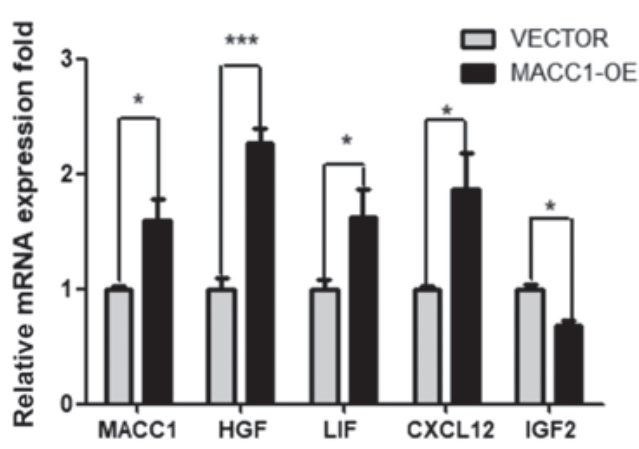

C

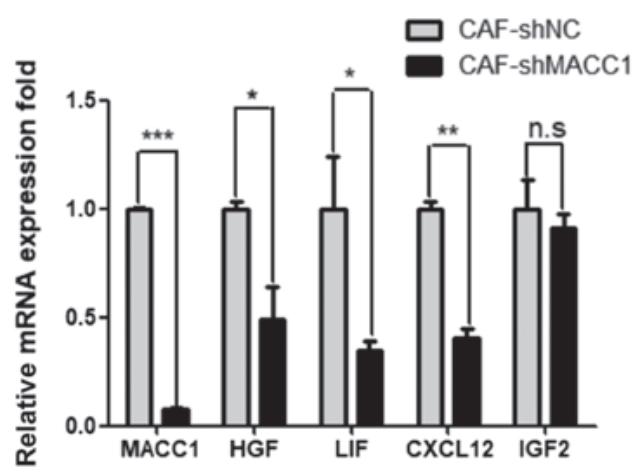

B

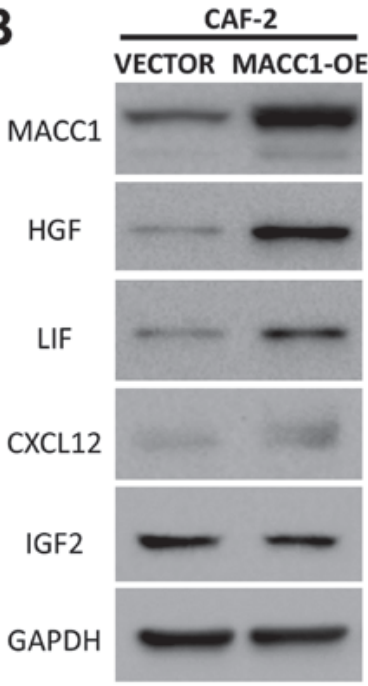

D

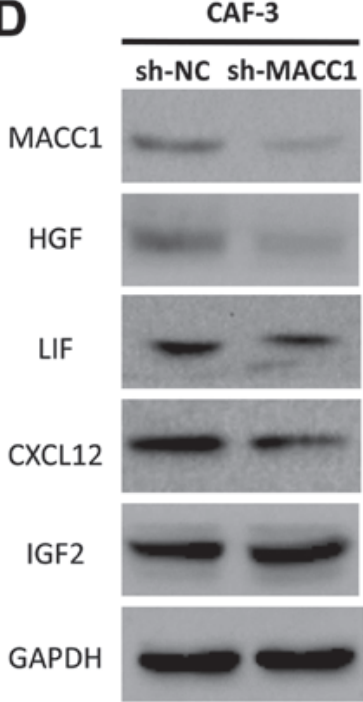

Figure 5. Overexpression of MACC1 induces CAFs to secrete chemokines through paracrine signaling. RT-qPCR analysis revealed the expression levels of HFG, LIF, CXCL12 and IGF2 in (A) MACC1-overexpressing CAF-2 cells and (B) shMACC1 CAF-3 cells. Western blot analysis revealed the expression level of HFG, LIF, CXCL12 and IGF2 in (C) MACC1-overexpressing CAF-2 cells and (D) shMACC1 CAF-3. Error bars represent the means \pm SD derived from 3 independent experiments. ${ }^{*} \mathrm{P}<0.05,{ }^{* *} \mathrm{P}<0.01$ and ${ }^{* * * *} \mathrm{P}<0.005$. MACC1, metastasis-associated in colon cancer- 1 ; CAFs, carcinoma-associated fibroblasts; HFG, hepatocyte growth factor; LIF, leukemia inhibitory factor; IGF2, insulin-like growth factor 2.

that the overexpression of MACC1 may stimulate CAFs to produce and secrete these chemokines via paracrine signaling, thus promoting lung adenocarcinoma cell invasion.

\section{Discussion}

The present study indicates a previously unrecognized role of MACC1 in CAFs, namely the promotion of the invasive ability of lung adenocarcinoma cells based on the following findings: i) CAFs existed in lung adenocarcinoma tissue; ii) CAFs induced the invasion of lung adenocarcinoma cells; iii) MACC1 overexpression promoted the invasive ability of CAFs; and iv) $\mathrm{MACCl}$ overexpression in CAFs stimulated the invasion of lung adenocarcinoma cells, and MACC1 overexpression induced CAFs to secrete chemokines through paracrine signaling.

Previous research hasidentified that the invasionand metastasis of various types of tumor are induced by CAFs, including lung adenocarcinoma $(15,26)$. It has become increasingly clear that
CAFs promote tumor progression (27). Activated fibroblasts induce epithelial cells, causing tumor invasion and metastasis, this effect is termed 'collective invasion' $(28,29)$. In this study, we constructed a microfluidic device to confirm that CAFs promoted the invasive ability of A549 cells (lung adenocarcinoma) in the 3D co-culture conditions.

It has been reported that MACC1 promotes the invasion of various types of cancer cells, proliferation and migration in cell culture and distant metastasis in mouse models $(16,30,31)$. The overexpression of MACC1 has been associated with a poor prognosis in various types of cancer (32-34). The inhibition of MACC1 expression has been demonstrated to repress tumor cell invasion and migration (35). Recently, we found that the activated MACC1/c-Met pathway, regulated by YB-1, promoted tumor proliferation and invasion in lung adenocarcinoma (36). CAFs and MACC1 both function in tumor progression, and we hypothesized that MACC1 may modulated CAF activity. In this study, we demonstrated that the local invasive capacity of CAFs 
was enhanced by the overexpression MACC1. Furthermore, the overexpression of MACC1 in CAFs increased the area and distance of lung adenocarcinoma cell invasion. MACC1 knockdown resulted in the reduced ability of CAFs to induce lung adenocarcinoma invasion.

Some researchers have demonstrated that CAFs can secrete extracellularmatrix components, growth factors and chemokines which promote tumor invasion and metastasis $(4,37)$. The crosstalk between tumor cells and stroma CAFs has also been indicated to contribute to tumor progression via HGF signaling (38). MACC1 was previously reported to regulate invasion and metastasis through regulating the $\mathrm{HGF} / \mathrm{c}-\mathrm{Met}$ pathway (16). Of note, in this study, we found that HGF, among other chemokines, was upregulated in MACC1-overexpressing CAFs. We thus hypothesized that MACC1 enhances paracrine signaling in $\mathrm{CAFs}$ and promotes lung adenocarcinoma cell invasion. However, further studies are required to focus on the effect of paracrine signaling inhibition on crosstalk between CAFs and lung adenocarcinoma.

In conclusion, in this study, we demonstrated that the overexpression of MACC1 in CAFs promoted the invasive ability of lung adenocarcinoma cells via paracrine signaling. The targeting of MACC1 may thus prove to be a potential therapeutic strategy for overcoming the CAF-induced invasion in lung adenocarcinoma.

\section{Acknowledgements}

The authors are thankful to Miss Qianqian Bi, Miss Mengying Yang, Mr. Zhe Sun, Mr. Qi Yang and Mr. Chao Li from Dalian Medical University for preparing the pathological specimens.

\section{Funding}

This study was supported by grants from the National Natural Science Foundation of China (nos. 81173453 and 81774078), and the Natural Science Foundation of Liaoning Province, China (nos. 201602227 and 20170540300).

\section{Availability of data and materials}

The datasets used and/or analyzed during the current study are available from the corresponding author on reasonable request.

\section{Authors' contributions}

TG, ZL, JL and CG conceived and designed the study. TG and ZL were involved in research administration. ZL, TG, LF, XW, NL, PW, SZ, FL, YC, XS and LZ performed the experiments and analyzed the data. TG, ZL, JL and CG wrote and edited the manuscript. All authors have read and approved the final manuscript.

\section{Ethics approval and consent to participate}

All experiments using human samples were approved by the Medical Ethical Committees of the First Affiliated Hospital of Dalian ethics committee Medical University (Ethics no. YJ-KY-FB-20). All patients signed written informed consent.

\section{Patient consent for publication}

Not applicable.

\section{Competing interests}

The authors declare that they have no competing interests.

\section{References}

1. Allemani C, Weir HK, Carreira H, Harewood R, Spika D, Wang XS, Bannon F, Ahn JV, Johnson CJ, Bonaventure A, et al; CONCORD Working Group: Global surveillance of cancer survival 1995-2009: Analysis of individual data for $25,676,887$ patients from 279 population-based registries in 67 countries (CONCORD-2). Lancet 385: 977-1010, 2015.

2. Langer CJ, Besse B, Gualberto A, Brambilla E and Soria JC: The evolving role of histology in the management of advanced non-small-cell lung cancer. J Clin Oncol 28: 5311-5320, 2010

3. Perlikos F, Harrington KJ and Syrigos KN: Key molecular mechanisms in lung cancer invasion and metastasis: A comprehensive review. Crit Rev Oncol Hematol 87: 1-11, 2013.

4. Kalluri R and Zeisberg M: Fibroblasts in cancer. Nat Rev Cancer 6: 392-401, 2006.

5. Gupta GP and Massagué J: Cancer metastasis: Building a framework. Cell 127: 679-695, 2006.

6. Shekhar MP, Pauley R and Heppner G: Host microenvironment in breast cancer development: Extracellular matrix-stromal cell contribution to neoplastic phenotype of epithelial cells in the breast. Breast Cancer Res 5: 130-135, 2003.

7. Olaso E, Salado C, Egilegor E, Gutierrez V, Santisteban A, Sancho-Bru P, Friedman SL and Vidal-Vanaclocha F: Proangiogenic role of tumor-activated hepatic stellate cells in experimental melanoma metastasis. Hepatology 37: 674-685, 2003.

8. Orimo A, GuptaPB,Sgroi DC,Arenzana-Seisdedos F,Delaunay T, Naeem R, Carey VJ, Richardson AL and Weinberg RA: Stromal fibroblasts present in invasive human breast carcinomas promote tumor growth and angiogenesis through elevated SDF-1/CXCL12 secretion. Cell 121: 335-348, 2005.

9. De Wever O and Mareel M: Role of tissue stroma in cancer cell invasion. J Pathol 200: 429-447, 2003.

10. Ostman A and Augsten M: Cancer-associated fibroblasts and tumor growth--bystanders turning into key players. Curr Opin Genet Dev 19: 67-73, 2009.

11. Xing F, Saidou J and Watabe K: Cancer associated fibroblasts (CAFs) in tumor microenvironment. Front Biosci 15: 166-179, 2010.

12. Unger C, Kramer N, Unterleuthner D, Scherzer M, Burian A, Rudisch A, Stadler M, Schlederer M, Lenhardt D, Riedl A, et al: Stromal-derived IGF2 promotes colon cancer progression via paracrine and autocrine mechanisms. Oncogene 36: 5341-5355, 2017.

13. Teng F, Tian WY, Wang YM, Zhang YF, Guo F, Zhao J, Gao C and Xue FX: Cancer-associated fibroblasts promote the progression of endometrial cancer via the SDF-1/CXCR4 axis. J Hematol Oncol 9: 8, 2016.

14. Kuzet SE and Gaggioli C: Fibroblast activation in cancer: When seed fertilizes soil. Cell Tissue Res 365: 607-619, 2016.

15. Neri S, Hashimoto H, Kii H, Watanabe H, Masutomi K, Kuwata T, Date H, Tsuboi M, Goto K, Ochiai A, et al: Cancer cell invasion driven by extracellular matrix remodeling is dependent on the properties of cancer-associated fibroblasts. J Cancer Res Clin Oncol 142: 437-446, 2016.

16. Stein U, Walther W, Arlt F, Schwabe H, Smith J, Fichtner I, Birchmeier W and Schlag PM: MACC1, a newly identified key regulator of HGF-MET signaling, predicts colon cancer metastasis. Nat Med 15: 59-67, 2009.

17. Arlt F and Stein U: Colon cancer metastasis: MACC1 and Met as metastatic pacemakers. Int J Biochem Cell Biol 41: 2356-2359, 2009.

18. Wang L, Wu Y, Lin L, Liu P, Huang H, Liao W, Zheng D, Zuo Q, Sun L, Huang N, et al: Metastasis-associated in colon cancer-1 upregulation predicts a poor prognosis of gastric cancer, and promotes tumor cell proliferation and invasion. Int J Cancer 133: 1419-1430, 2013. 
19. Huang Y, Zhang H, Cai J, Fang L, Wu J, Ye C, Zhu X and Li M: Overexpression of MACC1 and Its significance in human Breast Cancer Progression. Cell Biosci 3: 16, 2013.

20. Ji D, Lu ZT, Li YQ, Liang ZY, Zhang PF, Li C, Zhang JL, Zheng $\mathrm{X}$ and Yao YM: MACC1 expression correlates with PFKFB2 and survival in hepatocellular carcinoma. Asian Pac J Cancer Prev 15: 999-1003, 2014.

21. Gao J, Ding F, Liu Q and Yao Y: Knockdown of MACC1 expression suppressed hepatocellular carcinoma cell migration and invasion and inhibited expression of MMP2 and MMP9. Mol Cell Biochem 376: 21-32, 2013.

22. Shimokawa H, Uramoto H, Onitsuka T, Chundong G, Hanagiri T, Oyama T and Yasumoto K: Overexpression of MACC1 mRNA in lung adenocarcinoma is associated with postoperative recurrence. J Thorac Cardiovasc Surg 141: 895-898, 2011.

23. Chundong G, Uramoto H, Onitsuka T, Shimokawa H, Iwanami T, Nakagawa M, Oyama T and Tanaka F: Molecular diagnosis of MACC1 status in lung adenocarcinoma by immunohistochemical analysis. Anticancer Res 31: 1141-1145, 2011.

24. Guo T, Kong J, Liu Y, Li Z, Xia J, Zhang Y, Zhao S, Li F, Li J and Gu C: Transcriptional activation of NANOG by YBX1 promotes lung cancer stem-like properties and metastasis. Biochem Biophys Res Commun 487: 153-159, 2017.

25. Wang Z, Cai M, Weng Y, Zhang F, Meng D, Song J, Zhou H and Xie Z: Circulating MACC1 as a novel diagnostic and prognostic biomarker for nonsmall cell lung cancer. J Cancer Res Clin Oncol 141: 1353-1361, 2015.

26. Yu T, Guo Z, Fan H, Song J, Liu Y, Gao Z and Wang Q: Cancer-associated fibroblasts promote non-small cell lung cancer cell invasion by upregulation of glucose-regulated protein 78 (GRP78) expression in an integrated bionic microfluidic device. Oncotarget 7: 25593-25603, 2016.

27. Mueller MM and Fusenig NE: Friends or foes - bipolar effects of the tumour stroma in cancer. Nat Rev Cancer 4: 839-849, 2004.

28. Friedl P, Noble PB, Walton PA, Laird DW, Chauvin PJ, Tabah RJ, Black M and Zänker KS: Migration of coordinated cell clusters in mesenchymal and epithelial cancer explants in vitro. Cancer Res 55: 4557-4560, 1995.

29. Friedl P, Locker J, Sahai E and Segall JE: Classifying collective cancer cell invasion. Nat Cell Biol 14: 777-783, 2012.
30. Migliore C, Martin V, Leoni VP, Restivo A, Atzori L, Petrelli A, Isella C, Zorcolo L, Sarotto I, Casula G, et al: MiR-1 downregulation cooperates with MACC1 in promoting MET overexpression in human colon cancer. Clin Cancer Res 18: 737-747, 2012.

31. Galimi F, Torti D, Sassi F, Isella C, Corà D, Gastaldi S, Ribero D, Muratore A, Massucco P, Siatis D, et al: Genetic and expression analysis of MET, MACC1, and HGF in metastatic colorectal cancer: Response to met inhibition in patient xenografts and pathologic correlations. Clin Cancer Res 17: 3146-3156, 2011.

32. Chen S, Zong ZH, Wu DD, Sun KX, Liu BL and Zhao Y: The role of metastasis-associated in colon cancer 1 (MACC1) in endometrial carcinoma tumorigenesis and progression. Mol Carcinog 56: 1361-1371, 2017.

33. Zhou L, Yu L, Zhu B, Wu S, Song W, Gong X and Wang D: Metastasis-associated in colon cancer-1 and aldehyde dehydrogenase 1 are metastatic and prognostic biomarker for non-small cell lung cancer. BMC Cancer 16: 876, 2016.

34. Tan W, Xie X, Li L, Tang H, Ye X, Chen L, Tang W, Gao J, Pan L, Zhang X, et al: Diagnostic and prognostic value of serum MACC1 in breast cancer patients. Oncotarget 7: 84408-84415, 2016.

35. Zhang Y, Wang Z, Chen M, Peng L, Wang X, Ma Q, Ma F and Jiang B: MicroRNA-143 targets MACC1 to inhibit cell invasion and migration in colorectal cancer. Mol Cancer 11: 23, 2012.

36. Guo T, Zhao S, Wang P, Xue X, Zhang Y, Yang M, Li N, Li Z, $\mathrm{Xu} \mathrm{L}$, Jiang L, et al: YB-1 regulates tumor growth by promoting MACC1/c-Met pathway in human lung adenocarcinoma. Oncotarget 8: 48110-48125, 2017.

37. Gaggioli C, Hooper S, Hidalgo-Carcedo C, Grosse R, Marshall JF, Harrington K and Sahai E: Fibroblast-led collective invasion of carcinoma cells with differing roles for RhoGTPases in leading and following cells. Nat Cell Biol 9: 1392-1400, 2007.

38. Wu X, Chen X, Zhou Q, Li P, Yu B, Li J, Qu Y, Yan J, Yu Y, Yan M, et al: Hepatocyte growth factor activates tumor stromal fibroblasts to promote tumorigenesis in gastric cancer. Cancer Lett 335: 128-135, 2013. 